\title{
NEUTRINOS AND DARK MATTER
}

\author{
CHUNG-PEI MA \\ Department of Physics and Astronomy, University of Pennsylvania \\ Philadelphia, PA 19104 \\ E-mail: cpma@strad.physics.upenn.edu
}

\begin{abstract}
In these lectures I highlight some key features of massive neutrinos in the context of cosmology. I first review the thermal history and the free-streaming kinematics of the uniform cosmic background neutrinos. I then describe how fluctuations in the phase space distributions of neutrinos and other particles arise and evolve after neutrino decoupling according to the linear perturbation theory of gravitational instability. The different clustering properties of massive neutrinos (aka hot dark matter) and cold dark matter are contrasted. The last part discusses the nonlinear stage of gravitational clustering and highlights the effects of massive neutrinos on the formation of cosmological structure.
\end{abstract}

\section{Neutrino Masses}

These lectures discuss how the universe serves as a learning ground for massive neutrinos. Before doing so, let us briefly review some experimental measurements of neutrino masses.

Upper bounds on neutring masses from kinematic measurements in laboratories continue to improve. 1 For the $\tau$-neutrino, $m_{\nu_{\tau}}<18.2 \mathrm{MeV}$ from the decay channel $\tau \rightarrow 5 \pi+\nu_{\tau}$. For the $\mu$-neutrino, $m_{\nu_{\mu}}<170 \mathrm{keV}$ from twobody pion decay. For the electron neutrino, the quantity $m_{\nu_{e}}^{2}$ is measured in tritium beta decay by fitting the shape of the energy spectrum near the endpoint. Experiments thus far have yielded nonphysical negative values for $m_{\nu_{e}}^{2}$, indicating unexplained systematic effects in the measurements. A conservative upper bound is put at $m_{\nu_{e}} \approx 15 \mathrm{eV}$. The spread in arrival times of neutrinos from supernova explosions provides an independent way to constrain the mass of the electron neutrino. Various limits have been reported for SN 1987A; a conservative estimate is $m_{\nu_{e}}<23 \mathrm{eV}$. 证

\section{Properties of Cosmic Background Neutrinos}

\subsection{Temperature and Density}

For a brief 1 second after the big bang, neutrinos enjoy being part of the thermal bath composed of photons, electrons, protons, neutrons, and the associated anti-particles (after the quark-hadron era). The weak interactions at this early time are rapid enough to keep these particles in thermal equilibrium at a single temperature $T$. After 1 second, when $T$ drops below about 1 
$\mathrm{MeV}$, however, the neutrino interaction rate becomes slower than the Hubble expansion, and neutrinos become effectively collisionless and freely-streaming particles whose trajectories are determined by the geodesic equations. This event is commonly referred to as "neutrino decoupling." As the universe expands, the momenta and temperature of neutrinos are simply redshifted, and the neutrino temperature is given by the familiar formulas

$$
T_{\nu}(a)=a^{-1} T_{\nu, 0}, \quad T_{\nu, 0}=\left(\frac{4}{11}\right)^{1 / 3} T_{\gamma, 0}=1.947 K,
$$

where $a$ is the cosmic scale factor, the subscripts 0 denote the present-day values, and the cosmic background photon temperature is taken to be $T_{\gamma, 0}=$ $2.728 \mathrm{~K}$. 3

An important feature of the neutrino distribution after decoupling is that, although weak interactions are no longer rapid enough to keep neutrinos in thermal equilibrium with other particle species, neutrinos retain their equilibrium distribution as long as no other physical processes (e.g., gravitational clustering; see Sec. 3) are present to alter it. Therefore, to zeroth order in density and metric perturbations, the phase space distribution $f_{0}$ of the cosmic background neutrinos is of the simple Fermi-Dirac form

$$
f_{0}(\epsilon)=\frac{g_{s}}{h_{p}^{3}} \frac{1}{e^{\epsilon / k_{B} T_{\nu, 0}+1}},
$$

where $\epsilon=a\left(p^{2}+m_{\nu}^{2}\right)^{1 / 2}$ is the comoving energy, $T_{\nu, 0}$ is the neutrino temperature given by Eq. (1), $g_{s}$ is the number of spin degrees of freedom, and $h_{p}$ and $k_{B}$ are the Planck and the Boltzmann constants.

The situation is further simplified if neutrino masses are $\ll 1 \mathrm{MeV}$. Such neutrinos are highly relativistic at decoupling; their energy $\epsilon$, and hence the distribution function $f_{0}$, are independent of $m_{\nu}$ to a good approximation. One can easily show that, as long as $m_{\nu} \ll 1 \mathrm{MeV}$, the number density of the cosmic background neutrinos is related to the neutrino temperature by

$$
n_{\nu}\left(T_{\nu}\right)=\frac{7 g_{s}}{8 \pi^{2}} \zeta(3)\left(\frac{k_{B} T_{\nu}}{\hbar c}\right)^{3},
$$

where $\zeta(3) \approx 1.202$ is the Riemann zeta function of order 3 . This gives a present-day density of $\approx 113 \mathrm{~cm}^{-3}$ for every neutrino species independent of their masses. (For comparison, the present-day photon density is $\approx 412 \mathrm{~cm}^{-3}$.) It also follows that the contribution of these neutrinos to the present-day mass density parameter, $\Omega_{\nu}$, is related to their masses by the simple relation

$$
\Omega_{\nu} h^{2}=\frac{\Sigma_{i} m_{i}}{93 \mathrm{eV}}
$$


where the index $i$ runs over all light, stable neutrino species (e.g., $\nu_{e}, \nu_{\mu}$, and $\nu_{\tau}$ ), and the Hubble constant is $H_{0}=100 h \mathrm{~km} \mathrm{~s}^{-1} \mathrm{Mpc}^{-1}$. One then arrives at the important conclusion that in order for neutrinos not to close universe (i.e. $\Omega_{\nu} \leq 1$ ), the sum of neutrino masses must not exceed $93 h^{2} \mathrm{eV}$. This value is far below the current laboratory limits (see Sec. 1). Cowsik \& McClelland 1 were the first to use such cosmological arguments to place an upper bound on neutrino masses. (Unfortunately, these "hot dark matter" models in which the mass density is dominated by massive neutrinos have been found to produce excessive large voids surrounded by large coherent sheets and filaments that are not seen in the observable universe. 5 Modifications to this model will be discussed below.)

In the high mass regime, $m_{\nu} \gg 1 \mathrm{MeV}$, there exists another window where the neutrino contribution to the mass density parameter $\Omega$ of the universe is subcritical. The argument is that neutrinos with $m_{\nu} \gg 1 \mathrm{MeV}$ become nonrelativistic long before decoupling. Neutrino and anti-neutrino pairs cease to be created in abundance once the thermal temperature drops below $m_{\nu}$, and the neutrino density is suppressed by the Boltzmann factor $e^{-m_{\nu} / k_{B} T}$. This large reduction factor in the relic abundance allows neutrinos to have large masses without overclosing the universe. A more careful calculation 6 shows that an $\Omega \leq 1$ universe implies a lower limit of $\sim 2 \mathrm{GeV}$ if these heavy neutrinos are Dirac, and $\sim 6 \mathrm{GeV}$ if they are Majorana. Since this mass range is well above the current upper mass bounds from laboratory measurements, it is of interest only when one considers more exotic theories for neutrinos.

\subsection{Kinematics and Free Streaming}

Let us now turn to the kinematics and the streaming properties of neutrinos. In general, neutrinos of mass $m_{\nu}$ become non-relativistic after a redshift of

$$
z_{\mathrm{rel}} \approx \frac{m_{\nu} c^{2}}{3 k_{B} T_{\nu, 0}}=2 \times 10^{3}\left(\frac{m_{\nu}}{1 \mathrm{eV}}\right)
$$

This redshift has important implications for structure formation because it dictates the time at which massive neutrinos begin to make a transition from being radiation to matter. Note that this transition occurs fairly early, before

recombination if $m_{\nu} \gtrsim 1 \mathrm{eV}$. The average momentum of the cosmic background neutrinos at temperature $T_{\nu}$ is given by

$$
\langle p\rangle=3.15 k_{B} T_{\nu} / c .
$$


In the non-relativistic regime $\left(p=m_{\nu} v\right)$, the average neutrino speed can be written as

$$
\langle v\rangle=160 \mathrm{~km} / \mathrm{s}\left(\frac{1 \mathrm{eV}}{m_{\nu}}\right)\left(\frac{T_{\nu}}{1.947}\right) .
$$

Since $T_{\nu} \propto a^{-1} \propto(1+z)$, massive neutrinos slow down as time goes on. It is important to keep in mind that neutrinos with a mass of several $\mathrm{eV}$ have slowed down to an average velocity below $100 \mathrm{~km} \mathrm{~s}^{-1}$ today.

We also note that at the redshift of matter-radiation equality, $z_{\text {eq }} \sim$ $24000 \Omega h^{2}$ (i.e. when the total energy density in radiation in the universe equals that in matter), light neutrinos with $1<m_{\nu}<10 \mathrm{eV}$ are zooming around with speeds close to $c$. Such large thermal speeds prevent massive neutrinos from clustering gravitationally during this epoch, and this is why light neutrinos are referred to as hot dark matter (HDM). In contrast, perturbations in cold dark matter (CDM), which by definition has negligible thermal velocities, can grow unimpeded after $z_{\text {eq }}$. I will quantify the different clustering behavior of CDM and HDM further in Sec. 3 and 4.

Since neutrinos cannot cluster appreciably via gravitational instabilities on scales below the free streaming distance, this introduces a characteristic length scale into the problem. This scale is given by the free-streaming wavenumber (in comoving coordinates)

$$
k_{\mathrm{fs}}^{2}=\frac{4 \pi G \rho a^{2}}{\langle v\rangle^{2}},
$$

which is analogous to the Jeans length for a self-gravitating system of density $\rho$. For $k<k_{\mathrm{fs}}$ (i.e. large wavelengths), the density perturbation in the neutrinos is Jeans unstable and grows unimpeded in the matter-dominated era. For $k>k_{\mathrm{fs}}$, the density perturbation decays due to neutrino phase mixing. A phase-space interpretation of the free streaming property is that the phase mixing of collisionless particles damps the growth of density perturbations. 6 When the neutrinos are relativistic, $\langle v\rangle \approx c$, and the free-streaming distance is approximately the particle horizon, which scales as $k_{\mathrm{fs}}(a) \propto a^{-1}$ (in the radiation-dominated era). After the neutrinos become non-relativistic the relations $\langle v\rangle \propto a^{-1} m_{\nu}^{-1}, m_{\nu} \propto \Omega_{\nu} h^{2}$, and $\rho \propto a^{-3} h^{2}$ then imply 9

$$
k_{\mathrm{fs}}(a) \propto a^{1 / 2} \Omega_{\nu} h^{3} .
$$

As expected, the free-streaming distance $\left(\propto k_{\mathrm{fs}}^{-1}\right)$ decreases with time as the neutrinos slow down. This also implies that neutrinos can cluster gravitationally on increasingly small length scales at later times. 9 Such behavior has been seen in cosmological numerical simulations and will be discussed in Sec. 4. 


\section{Linear Perturbations in Neutrinos and Other Particles}

Thus far our discussion has focused on the properties of the smooth cosmic background neutrinos, and Eqs. (1)-(7) were derived under this assumption. The universe today, however, is clearly far from being homogeneous on scales of $\sim 100 \mathrm{Mpc}$ and below. Baryons and dark matter in galaxies, clusters, and superclusters show a wide spectrum of overdensities above the cosmic mean. The current theoretical framework for the origin and evolution of these cosmic structures rests upon the assumption that certain primordial fluctuations (perhaps originated from quantum fluctuations of scalar fields during the inflationary era) imprint a perturbation spectrum on all matter and radiation. These fluctuations subsequently grow via gravitational instabilities to give rise to the wide range of observed structures. How are the cosmic relic neutrinos affected by all this?

To understand the growth of density perturbations in neutrinos as well as other forms of matter and radiation, one would need to learn the linear cosmological perturbation theory of gravitational instability. A full description of this theory requires more time than is allocated for these lectures. I will only sketch the theory below with emphasis on the neutrino component. Interested readers should refer to the pioneering work of Lifshitz, later reviewed in Lifshitz \& Khalatnikov. 10 More modern treatments of various aspects of this theory can be found in the textbooks by Weinberg and Peebles, 11 in the reviews by Kodama \& Sasaki and Mukhanov, Feldman \& Brandenberger, 12 and in the Summer School lectures by Efstathiou, Bertschinger, and Bond. 13 A complete description of this theory for all relevant particles is given by Ma \& Bertschinger.14 Here, I will only discuss the essence of the theory and highlight the physical meaning of the key results.

\subsection{Neutrino Phase Space}

Let us start with the neutrinos. The full phase space distribution function of neutrinos can be written as

$$
f(\vec{x}, \vec{p}, t)=f_{0}(p)+f_{1}(\vec{x}, \vec{p}, t),
$$

where $f_{1}$ denotes perturbations to the Fermi-Dirac distribution $f_{0}$ given by Eq. (2). Unlike the unperturbed term $f_{0}$ that depends only on $p, f_{1}$ can have complicated dependence on time as well as positions $\vec{x}$ and the conjugate momenta $\vec{p}$. The equations for neutrino temperature, number density etc. discussed in Sec. 2 were obtained assuming $f=f_{0}$. A non-vanishing $f_{1}$ would lead to perturbations in these quantities. For example, the perturbed neutrino 
energy density is related to $f_{1}$ by

$$
\delta \rho(\vec{x}, t)=a^{-4} \int d^{3} p \epsilon f_{1}(\vec{x}, \vec{p}, t) .
$$

Other quantities such as perturbations in the pressure and shear can also be related to $f_{1}$.

It is in general difficult to compute and sample $f_{1}$ directly because at a given time, it depends on six variables. A Monte Carlo technique, or a "general-relativistic $N$-body" technique, has been developed to evolve $f_{1}$ from redshift $z \sim 10^{9}$ shortly after neutrino decoupling until $z \sim 10$ when nonlinear effects become non-negligible. 15 In this calculation, an ensemble of neutrino simulation particles is initially assigned velocities drawn from the Fermi-Dirac distribution, which is an excellent approximation at $z \sim 10^{9}$. The trajectory of each neutrino simulation particle is then followed by integrating the geodesic equations in the perturbed background spacetime. The metric perturbation gives rise to a nonzero $f_{1}$, and the particle positions and velocities at a later time $t$ represent a realization of $f_{1}(\vec{x}, \vec{p}, t)$. Results from this calculation have revealed that at $z \sim 15$, positive correlations have developed in the rms neutrino velocities and the overdensity, which would be absent if the phase-space distribution were purely Fermi-Dirac (i.e. $f=f_{0}$ ). The more spatially clustered neutrinos are found to move faster, possibly resulting from an increase in the kinetic energy during gravitational infalls.

\subsection{Evolution of Perturbed Density Fields}

The phase-space description above is applicable to all particle species and is used in the full theory. The full, general-relativistic version of the linear perturbation theory is described by a set of coupled and linearized Einstein, Boltzmann, and fluid equations. The variables include the metric perturbations to the homogeneous and isotropic Friedmann-Robertson-Walker spacetime, and the phase-space perturbations in all relevant particle species (e.g., photons, baryons, cold dark matter, massless and massive neutrinos). The Einstein equations describe how the time evolution of the metric perturbations is affected by the perturbations in the density, pressure, shear, and higher-order moments of matter and radiation. The Boltzmann and fluid equations, on the other hand, describe the time evolution of the radiation and matter distribution in the perturbed spacetime. Together, this theory describes the growth of metric and density perturbations throughout the early history of the universe, and it serves as the foundation for all calculations of the linear power spectra for matter and temperature variations imprinted on the cosmic microwave background. 


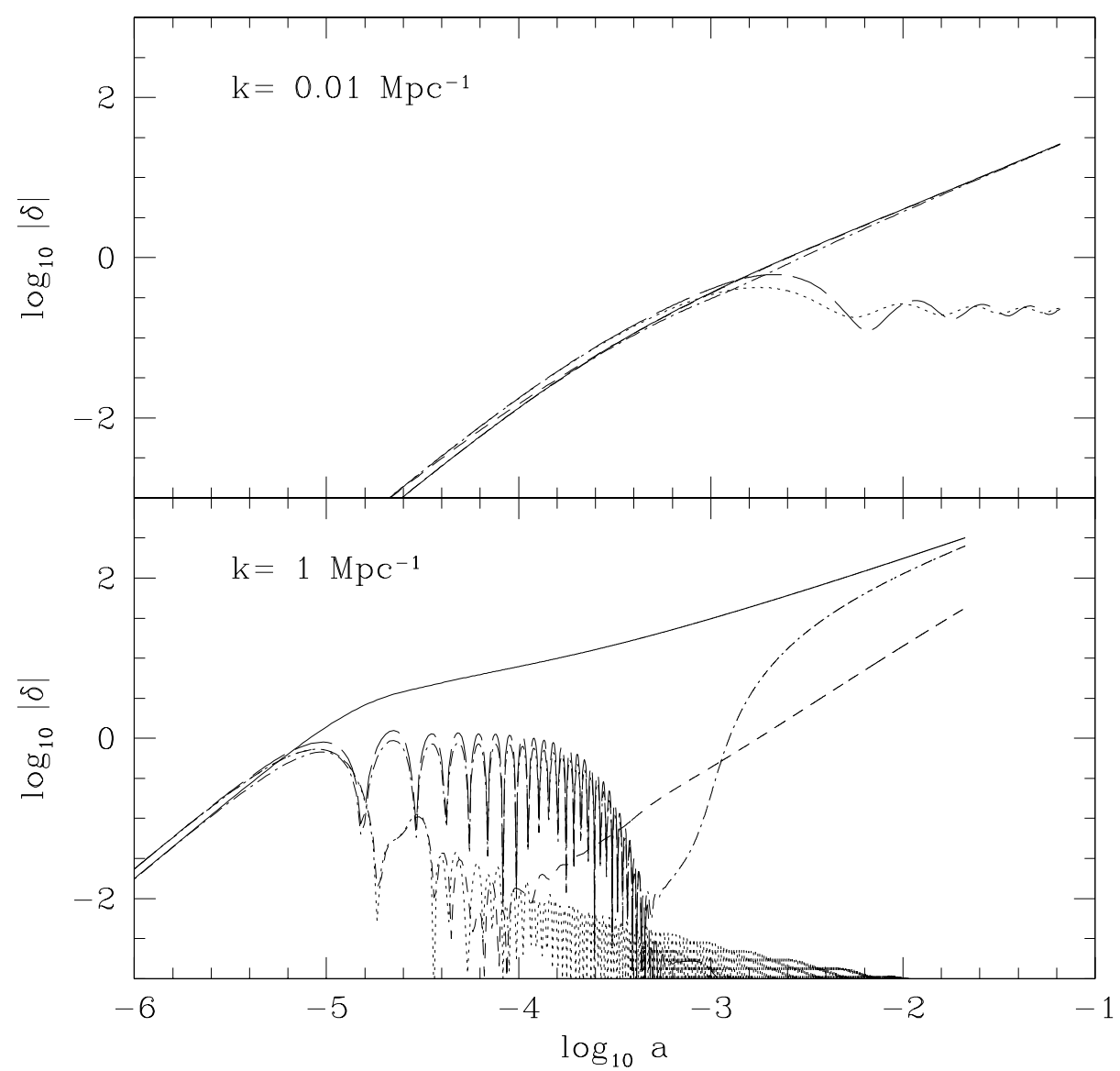

Figure 1: Time evolution of the perturbed energy density field, $\delta=\rho / \bar{\rho}-1$, for five matter and radiation components in a flat $\mathrm{C}+\mathrm{HDM}$ cosmological model. (See text for model parameters.) The results are from integration of the coupled Einstein and Boltzmann equations. Since the equations are linearized, each $k$-mode evolves independently. Two modes are shown here for illustration. In each panel, the five curves represent $\delta$ for the cold dark matter (solid), baryons (dash-dotted), photons (long-dashed), massless neutrinos (dotted), and massive neutrinos (short-dashed), respectively. 
Figure 11 illustrates a small subset of results that can be obtained from numerical integration of these linearized equations. It shows the time evolution of the perturbed energy density field, $\delta=\delta \rho / \bar{\rho}=\rho / \bar{\rho}-1$, for the five relevant particle species in a cold + hot dark matter $(\mathrm{C}+\mathrm{HDM})$ model. This model assumes an Einstein-de Sitter universe containing a mixture of CDM, HDM (i.e. massive neutrinos), baryons, photons, and massless neutrinos. The density parameters in the first three components in this model are $\Omega_{c}=0.75, \Omega_{\nu}=$ 0.2 , and $\Omega_{\mathrm{b}}=0.05$, and the Hubble parameter is taken to be $H_{0}=50 \mathrm{~km}$ $\mathrm{s}^{-1} \mathrm{Mpc}^{-1}$, or $h=0.5$. From Eq. (4), these parameters correspond to a neutrino mass of $4.7 \mathrm{eV}$. (For definiteness, this calculation has assumed that only one type of neutrinos, presumably $\nu_{\tau}$, has a non-negligible mass.) Two wavenumbers, $k=0.01 \mathrm{Mpc}^{-1}$ (top) and $1 \mathrm{Mpc}^{-1}$ (bottom), are shown in Figure 11 to demonstrate the intricate dependence of $\delta$ on length and time scales. The overall normalization of $\delta$ is set arbitrarily.

We observe several salient features in Figure 1. First, the amplitudes of $\delta$ for all particles grow monotonically until a critical time, after which different particle species exhibit very different behavior. This critical time is the "horizon crossing" time, and it occurs when the horizon has grown large enough to encompass the wavelength of a given mode of perturbation. A mode of perturbation is therefore not in causal contact until horizon crossing. Naturally, this occurs earlier for smaller wavelengths (i.e. larger $k$ ). In Figure 11, one can indeed see that the $k=1 \mathrm{Mpc}^{-1}$ mode enters the horizon at $a \sim 10^{-5}$ while the $k=0.01 \mathrm{Mpc}^{-1}$ mode enters the horizon a little after $a=10^{-3}$. A point to keep in mind is that the behavior of $\delta$ before horizon crossing is strongly dependent on the choice of gauge. The results shown in Figure 1 are computed in the so-called synchronous gauge, which is a popular choice due to historical precedent. 10 See Mukhanov, Feldman \& Brandenberger 12 and Ma \& Bertschinger 14 for discussion of a more convenient gauge (the conformal Newtonian gauge).

The second feature in Figure 1 to note is that after horizon crossing, the photons (long-dashed) and baryons (dot-dashed) exhibit rapid, coupled oscillations in the $k=1 \mathrm{Mpc}^{-1}$ mode but only the photons oscillate in the $k=0.01$ $\mathrm{Mpc}^{-1}$ mode. This occurs because the former enters the horizon before recombination at $a_{\text {rec }} \sim 10^{-3}$, and the photons and baryons are coupled by Thomson scattering and oscillate acoustically. The coupling is not perfect. The friction of the photons dragging against the baryons leads to Silk damping, 16 which is prominent in the bottom panel of Figure 11 at $a \sim 10^{-3.5}$. After recombination, the baryons decouple from the photons and fall quickly into the potential wells formed earlier by the CDM. This results in the rapid growth of the dotdashed curve in the bottom panel of Figures 1. The mode with $k=0.01$ 
$\mathrm{Mpc}^{-1}$, on the other hand, enters the horizon when the universe is neutral. The baryons therefore grow like the CDM and do not oscillate. The critical length scale demarcating these two regimes is the horizon size at recombination $a_{\text {rec }}: k_{\text {rec }} \sim 0.03 \mathrm{Mpc}^{-1}$.

The third feature to note in Figure 11 is the rate of growth of the CDM component (solid curve) after horizon crossing. Close inspection shows that the CDM in the bottom panel grows more slowly at $10^{-5} \lesssim a \lesssim 10^{-4}$ than later on, whereas in the upper panel, the CDM simply grows with a power law after horizon crossing. This is because the shorter wavelength mode $\left(k=1 \mathrm{Mpc}^{-1}\right)$ enters the horizon when the energy density of the universe is dominated by radiation, and fluctuations in matter (e.g. CDM) cannot grow appreciably during this era. The critical scale separating continual and suppressed growth is the horizon size at the time of radiation-matter equality $a_{\mathrm{eq}} \sim 4 \times 10^{-5}\left(\Omega h^{2}\right)^{-1}$ : $k_{\mathrm{eq}} \sim 0.1 \mathrm{Mpc}^{-1}$ for the parameters of this model.

The fourth feature to note in Figure 1 is the behavior of the massive neutrinos (short-dashed). As discussed in Sec. 2, neutrinos of masses within the cosmologically interesting range $(\sim 1$ to $10 \mathrm{eV})$ are highly non-relativistic today but were relativistic at earlier times. This property is in fact evident in the top panel of Figure 1: Careful inspection shows that at $a \approx 10^{-4}$, the density field $\delta$ in massive neutrinos is indeed making a gradual transition from the upper line for the radiation fields to the lower line for the matter fields. (More precisely, the primordial perturbations are assumed to be "isentropic" here, which leads to a perturbation amplitude that is a factor of $4 / 3$ higher for radiation than matter.) The subsequent evolution of $\delta$ in massive neutrinos for this mode $\left(k=0.01 \mathrm{Mpc}^{-1}\right)$ is very similar to that of CDM. This is because it enters the horizon when the thermal velocities of the neutrinos have decreased substantially; the free-streaming effect is therefore unimportant. For the $k=1$ $\mathrm{Mpc}^{-1}$ mode, on the other hand, the free streaming effect is evident and the growth of $\delta$ in massive neutrinos is suppressed until the characteristic freestreaming scale $k_{\mathrm{fs}}(a)$ given by Eq. (9) grows to $\sim k$. Afterwards, the shortdashed curve for massive neutrinos is seen to grow again and catch up to the CDM. Since $k_{\mathrm{fs}} \propto a^{1 / 2}$, the larger $k$ modes suffer more free-streaming damping and $\delta$ for massive neutrinos can not grow until later times. The damping in the massive neutrino component also affects the growth of the CDM, slowing it down more for models with larger $\Omega_{\nu}$ compared to the pure CDM model.

\subsection{Growth Rate and Power Spectrum}

I have used Figure 11 computed for two particular $k$-modes in a particular cosmological model to illustrate the physical meaning of many key features 
in the evolution of the density field for matter and radiation throughout the cosmic history. I will now discuss general descriptions that can be conveniently used to characterize the fluctuation amplitudes over a range of length scales for a variety of cosmological models. For example, it is extremely useful to know the dependence of $\delta$ and its time derivative on $k$ at a given time for a wide range of models. The most basic quantity to use is the linear power spectrum, $P(k, t)$, and the growth rate of the density field, $f \equiv d \log \delta / d \log a$. (Note: I am following the convention of using $f$ to denote the growth rate; it should not be confused with the phase-space distribution function of Sec. 2 and 3.1.) The power spectrum quantifies the two-point statistics of $\delta$, and for a Gaussian field, $P(k)$ represents its rms fluctuations and completely specifies its statistical properties. The power spectrum is therefore of fundamental importance in cosmology.

Comparing the growth rate and power spectrum for models with and without massive neutrinos is an effective way to illustrate the effects of hot dark matter. In the standard CDM model with $\Omega=1$ and $h=0.5$ (neutrino mass is assumed to be zero), the CDM density field grows as the expansion factor $a$ on all scales; therefore $f=1$. As discussed in Sec. 2.2, massive neutrinos introduce an additional length scale, the free-streaming distance, below which fluctuations are washed out and the growth rate is retarded. The growth rate is therefore more intricate in models with massive neutrinos and is generally a function of the wavenumber $k$, neutrino density parameter $\Omega_{\nu}$, and time. Figure 2 illustrates such dependence in four different $\mathrm{C}+\mathrm{HDM}$ models that assume a mixture of CDM and HDM. It shows that the growth is suppressed at large $k$, and models with a larger fraction of energy density in HDM suffer more. It also shows that the suppression becomes less severe at later times.

One can gain some understanding of the behavior shown in Figure 2 by exploring two asymptotic regions that can be solved analytically for $\mathrm{C}+\mathrm{HDM}$ models with $\Omega_{c}+\Omega_{\nu}=1$ : (1) Since HDM behaves like CDM above the freestreaming distance, $f \rightarrow 1$ as $k \rightarrow 0$; (2) In the opposite limit of large $k$, the HDM density field $\delta_{h}$ is severely dampened compared to the CDM density field $\delta_{c}$ because of the neutrino effects. For $\delta_{h} \ll \delta_{c}$, the time evolution of the CDM density field is governed by the linearized fluid equation

$$
\ddot{\delta_{c}}+\frac{\dot{a}}{a} \dot{\delta}_{c}=1.5 H^{2} a^{2} \Omega_{c} \delta_{c}
$$

where the dots denote differentiation with respect to the conformal time $\tau$. Since $H a=2 / \tau$ in the matter-dominated era, the growing solution in this 


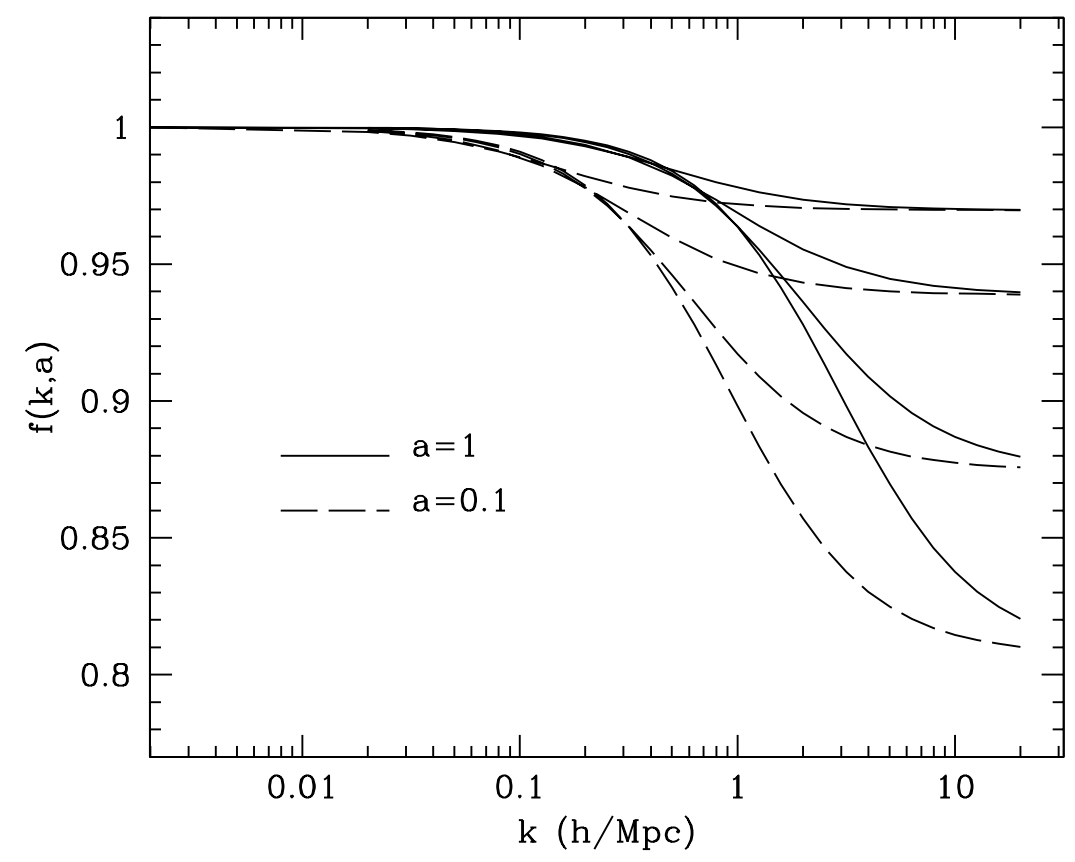

Figure 2: Growth rate of the CDM density field, $f \equiv d \log \delta / d \log a$, in four flat C+HDM models at $a=1$ (solid) and 0.1 (dashed). The four models assume different neutrino masses: $m_{\nu}=1.2,2.3,4.6$, and $6.9 \mathrm{eV}$ (from top down), corresponding to $\Omega_{\nu}=0.05,0.1,0.2$, and 0.3. At small $k$, the CDM density field in these models grows with the same rate $(\delta \propto a)$ as in the standard CDM model. At large $k$, the growth rate is suppressed because a fraction of the energy density in $\mathrm{C}+\mathrm{HDM}$ models is in the hot neutrinos that exhibit less gravitational clustering. The suppression at large $k$ becomes less severe at later times because the velocities of the hot neutrinos decrease with time. 
regime is easily shown to be 17

$$
f_{\infty} \equiv f(k \rightarrow \infty)=\frac{1}{4} \sqrt{1+24 \Omega_{c}}-\frac{1}{4}=\frac{5}{4} \sqrt{1-\frac{24}{25} \Omega_{\nu}}-\frac{1}{4} .
$$

It is interesting to note that

$$
f_{\infty} \approx \Omega_{c}^{0.6}
$$

is an excellent approximation to the equation above, especially for the cosmologically viable range of $\Omega_{\nu} \lesssim 0.3$. Using these analytic solutions in the asymptotic regimes and the scaling dependence of the free streaming wavenumber $k_{\mathrm{fs}}$ in Eq. (9), one can construct a simple approximation for $f$ for a wide range of model parameters. It is found that the growth rate $f$ is well approximated by 9

$$
f \equiv \frac{d \log \delta}{d \log a}=\frac{1+\Omega_{c}^{0.6} 0.00681 x^{1.363}}{1+0.00681 x^{1.363}}, \quad x \equiv \frac{k}{\Gamma_{\nu} h},
$$

where $\Gamma_{\nu}$ is a shape parameter derived from Eq. (9),

$$
\Gamma_{\nu}=a^{1 / 2} \Omega_{\nu} h^{2},
$$

$\Omega_{c}+\Omega_{\nu}=1$, and $k$ is in units of $\mathrm{Mpc}^{-1}$. Note that Eq. (15) depends only on the variable $x$ that characterizes the neutrino free-streaming scale, and $\Omega_{\nu}$ (or $\Omega_{c}$ ) via $f_{\infty}$. The fractional error of the fit relative to the numerically computed values is smaller than $0.5 \%$ for a wide range of parameters. The seemingly complicated multi-parameter dependence of Figure 2 is succinctly incorporated in Eq. (15).

For the linear power spectrum, the slower time growth of $\delta$ at $k>k_{\mathrm{fs}}$ for the $\mathrm{C}+\mathrm{HDM}$ models shown in Figure 2 indicates a suppressed clustering amplitude on these scales. This effect is illustrated in Figure 3, which shows the density-averaged power spectrum (i.e. $P=\left\{\Omega_{c} \sqrt{P_{c}}+\Omega_{\nu} \sqrt{P_{\nu}}\right\}^{2}$ ) for three flat $\mathrm{C}+\mathrm{HDM}$ models. In general, $P_{\nu} \ll P_{c}$ on small length scales due to the neutrino thermal velocities, and the models with higher $\Omega_{\nu}$ clearly have less power at large $k$ in accordance with Figure 2. A good approximation for the relative $P(k)$ in a flat $\mathrm{C}+\mathrm{HDM}$ model (with $\Omega_{\nu} \lesssim 0.3$ ) and a pure CDM model $\left(\Omega_{\nu}=0\right)$ is given by

$$
\begin{aligned}
& \frac{P\left(k, a, \Omega_{\nu}\right)}{P\left(k, a, \Omega_{\nu}=0\right)}=\left(\frac{1+b_{1} x^{b_{4} / 2}+b_{2} x^{b_{4}}}{1+b_{3} x_{0}^{b_{4}}}\right)^{\Omega_{\nu}^{1.05}}, \\
& x=\frac{k}{\Gamma_{\nu}}, \quad x_{0}=x(a=1),
\end{aligned}
$$




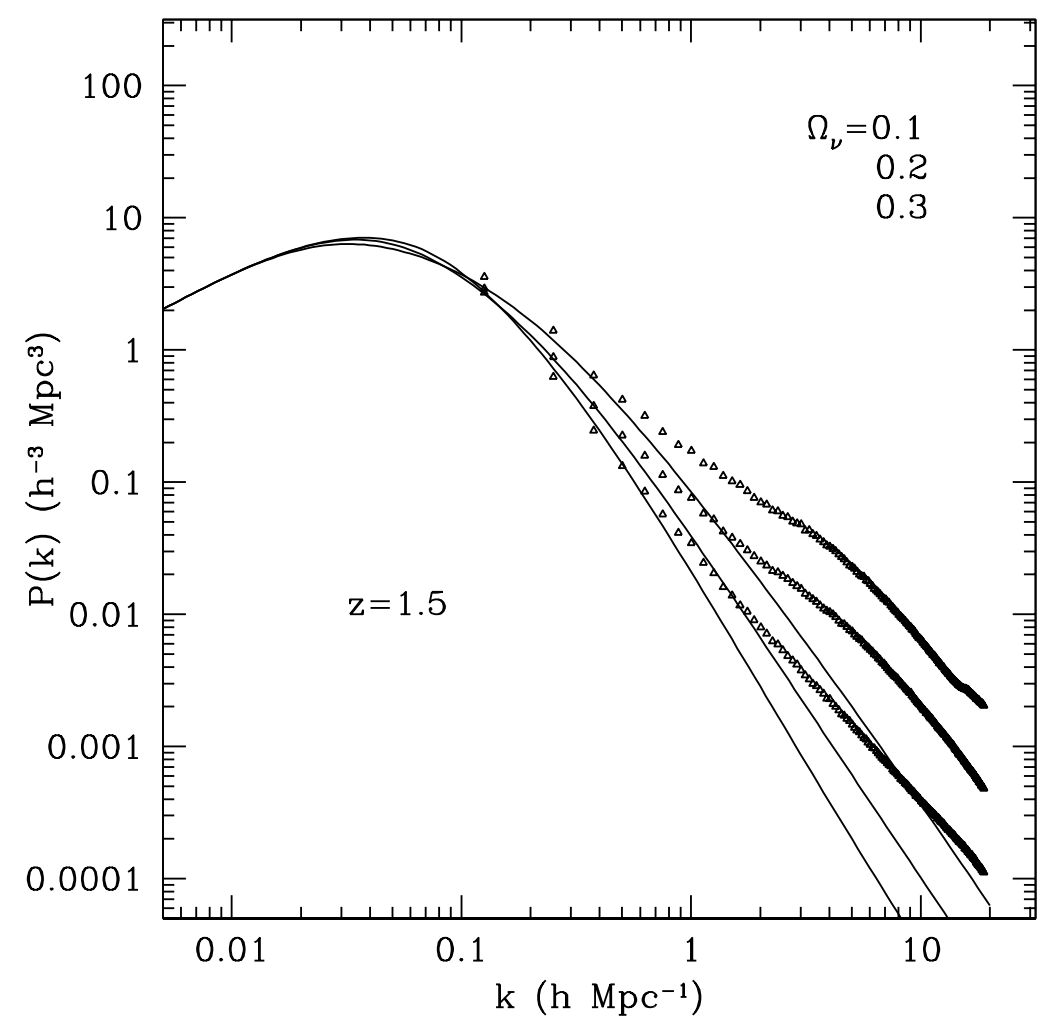

Figure 3: Power spectrum at $z=1.5$ for the perturbed matter density field for three flat C+HDM models with $\Omega_{\nu}=0.1,0.2$, and 0.3 . The solid curves show the linear $P(k)$ computed from the linear perturbation theory; accurate analytical fitting formulas are given by Eq. (17). The triangles show the nonlinear $P(k)$ computed from $N$-body simulations; accurate analytical approximations are given by Eq. (18). 
where the best-fit parameters are $b_{1}=0.004321, b_{2}=2.217 \times 10^{-6}, b_{3}=11.63$, and $b_{4}=3.317$ for $k$ in units of $\mathrm{Mpc}^{-1}$. Analytical approximations for the separate cold and hot spectra $P_{c}$ and $P_{\nu}$ can be found in the same reference. More complicated approximations for a wider range of parameter space have also been proposed.18

\section{Nonlinear Gravitational Clustering of Neutrinos}

As far as cosmological structure formation is concerned, the main difference between massive and massless neutrinos is that the former can participate in the processes of gravitational clustering and hence serves as a component of the dark matter in the universe. Massless neutrinos, on the other hand, affect cosmology only through their contribution to the radiation energy density. Any primordial perturbations in massless neutrinos are damped out after horizon crossing as a result of phase mixing (see dotted curves in Figure 11), and the only remnant of this component is the elusive $T_{\nu, 0}=1.947 \mathrm{~K}$ background described in Sec. 2.

\subsection{Spatial Distribution of Neutrinos}

For massive neutrinos in models with a mixture of CDM and HDM, it is interesting to ask: do the neutrinos fall in the CDM potential wells and form a part of dark matter halos? One may naively think not because neutrinos are too hot. Our discussion thus far, however, indicates otherwise. We have seen in Eq. (7) that neutrinos slow down with the expansion of the universe. Those with a mass of several $\mathrm{eV}$ are travelling with a speed much below the typical velocity dispersions $\sim 200 \mathrm{~km} \mathrm{~s}^{-1}$ of stars in galaxies. They can potentially be bound to galactic halos. In general, the extent to which massive neutrinos can cluster gravitationally depends on their mass and speed.

To illustrate this point further, let us examine results from numerical simulations. Figure 4 shows the projected spatial distribution of cold particles (middle), hot particles (bottom), and the sum of the two (top) in a simulated dark matter halo in a flat $\Omega_{\nu}=0.2 \mathrm{C}+\mathrm{HDM}$ model $\left(m_{\nu}=4.7 \mathrm{eV}\right)$. Three redshifts are shown (from left to right): $z=2,1$ and 0 when the universe is 2.5, 4.6, and 13 billion years old, respectively. Each panel is $3.5 \times 3.5 h^{-1} \mathrm{Mpc}$ in physical coordinates. The parent simulation is a large $N$-body run with 23 million simulation particles in a $(100 \mathrm{Mpc})^{3}$ comoving box. 19 The dense halo shown at $z=0$ is clearly formed from mergers of two smaller halos and their satellites at higher redshifts, demonstrating the "bottom-up" hierarchical pattern of structure formation which is preserved in $\mathrm{C}+\mathrm{HDM}$ models with $\Omega_{c}>\Omega_{\nu}$. Massive neutrinos are visibly clustered in the bottom panels, but their spatial 


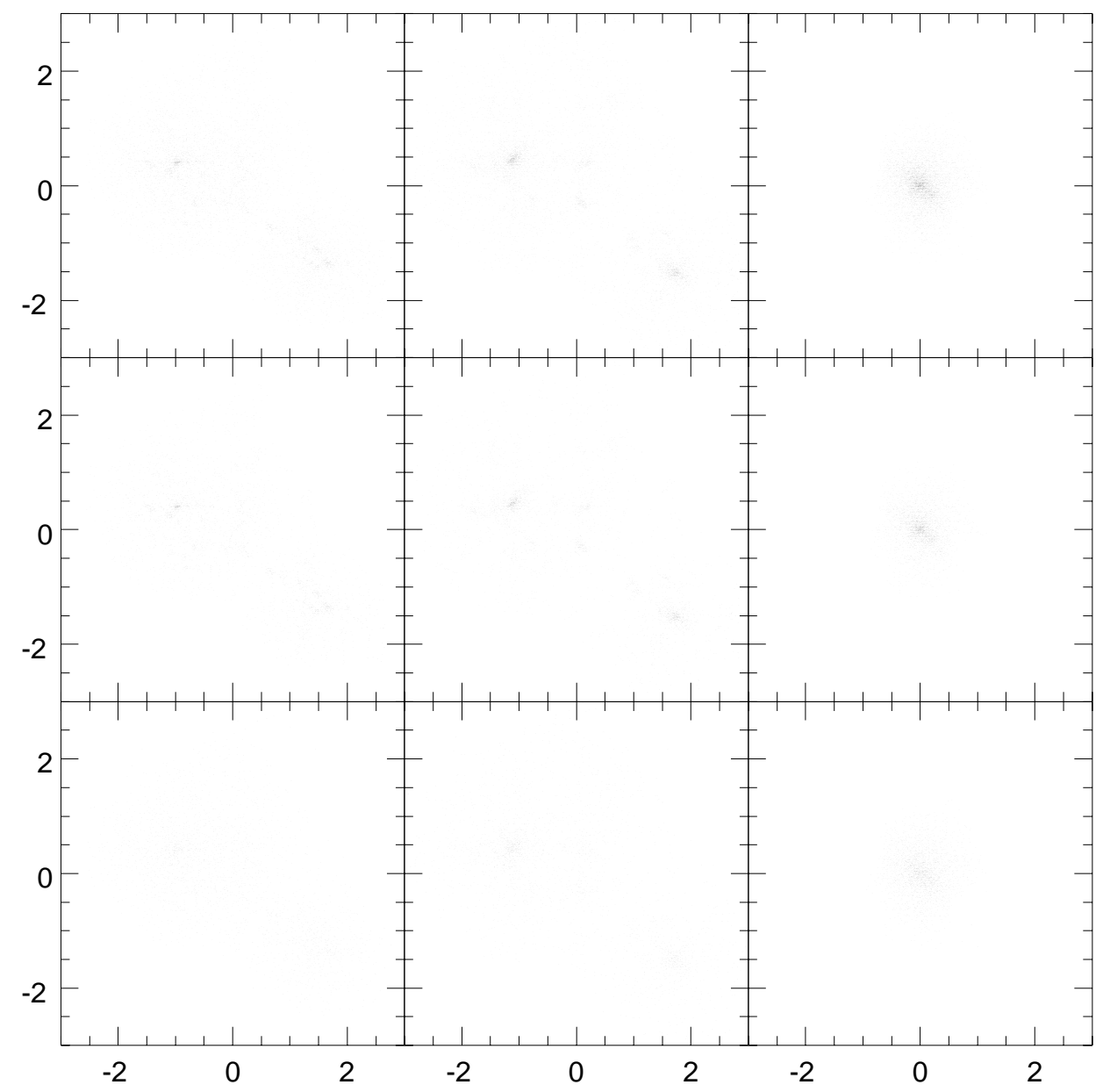

Figure 4: Spatial distribution of cold dark matter (middle) vs. hot dark matter (bottom) particles in a simulated halo formed in a large $N$-body run for the flat $\Omega_{\nu}=0.2 \mathrm{C}+\mathrm{HDM}$ model. The top panels shows the sum of cold and hot particles. Three redshifts are shown: $z=2,1$, and 0 (from left to right), corresponding to cosmic times of $2.5,4.6$, and 13 billion years. All boxes have the same physical scale, $3.5 \times 3.5 h^{-1} \mathrm{Mpc}$. The final halo at $z=0$ is clearly a merger product of two dominant subhalos and several smaller satellites at higher redshifts. The spatial distribution of the massive neutrinos $(4.7 \mathrm{eV})$ is visibly smoother than that of the CDM. 
distribution is smoother than that of the CDM. In the smaller CDM clumps at $z \gtrsim 1$, there are no discernible HDM halos at the same locations. The average thermal speed of an ensemble of $4.7 \mathrm{eV}$ Fermi-Dirac neutrinos at $z \gtrsim 1$ is $\gtrsim 70$ km/s (cf. Eq. 7). As discussed in Sec. 3.1, when linear perturbations are taken into account, neutrinos in overdense regions have even higher velocities. It is therefore not surprising that the shallower potential wells of these small halos cannot trap a substantial number of HDM particles.

The clustering of the $4.7 \mathrm{eV}$ neutrinos shown in Figure 4 should be compared with the constraint derived by Tremaine \& Gunn, 20 which states that if cosmic neutrinos were to make up the bulk of galactic and cluster halos, they must be more massive than $\sim 10 \mathrm{eV}$ so that the Pauli exclusion principle is not violated. The $\mathrm{C}+\mathrm{HDM}$ models considered here assume $\Omega_{c}>\Omega_{\nu}$ so as to preserve the successful hierarchical formation of structure in pure CDM models. Dark matter halos such as in Figure 1 have a substantial fraction of CDM, which in turn enhances the gravitational infalls of massive neutrinos. The clustering of neutrinos in $\mathrm{C}+\mathrm{HDM}$ models is therefore more complicated.

\subsection{Nonlinear Power Spectrum}

The process of nonlinear gravitational clustering can be quantified statistically. Here I will only discuss the lowest-order statistical description given by the nonlinear power spectrum $P(k)$ of the matter density field.

We have already discussed the linear power spectrum in Sec. 3.3. The triangles in Figure 3 show the nonlinear $P(k)$ computed from the particle positions in numerical simulations of three $\mathrm{C}+\mathrm{HDM}$ models. The hierarchical nature of gravitational collapse in these models is illustrated by the fact that the high- $k$ modes have become strongly nonlinear whereas the low- $k$ modes are still following the linear power spectrum. The fact that the lowest several $k$ modes are still linear at $z=0$ ensures that our choice of the simulation box size $(100 \mathrm{Mpc})$ is large enough to include all waves that have become nonlinear at present.

The calculation of the fully evolved $P(k)$ for a given model is a laborious task involving the execution of high-resolution $N$-body simulations. Fortunately, some recent progress has been made in constructing analytical fitting formulas for a wide range of interesting models. The strategy is to examine the mapping between the linear and nonlinear $P(k)$ for a small, selected set of models with $N$-body data and then to extract systematic behavior for a wider range of parameters. The work carried outhus far has investigated scale-free models with a power-law power spectrum 21,22 pure CDM and CDM with a cosmological constant $\Lambda$ (LCDM) models, 22.2324 and C+HDM models. 24 The proposed 
formulas typically have a functional form that is motivated by analytical solutions in asymptotic regimes, but in order to obtain accurate approximations, the coefficients are calculated from fits to the nonlinear $P(k)$ computed from the numerical simulations. These approximations have provided physical insight into the process of nonlinear collapse and much practical convenience in incorporating the prominent nonlinear effects illustrated in Figure 3.

The formula applicable for the widest range of cosmological models thus far is given below. It maps the density variance $\Delta(k) \equiv 4 \pi k^{3} P(k)$ in the linear and nonlinear regimes by 24

$$
\begin{aligned}
& \frac{\Delta_{\mathrm{nl}}(k)}{\Delta_{\mathrm{l}}\left(k_{0}\right)}=G\left(\frac{\Delta_{\mathrm{l}}\left(k_{0}\right)}{g_{0}^{1.5} \sigma_{8}^{\beta}}\right), \\
& G(x)=[1+\ln (1+0.5 x)] \frac{1+0.02 x^{4}+c_{1} x^{8} / g^{3}}{1+c_{2} x^{7.5}} .
\end{aligned}
$$

Note that $\Delta_{1}$ and $\Delta_{\mathrm{nl}}$ are evaluated at different wavenumbers, where $k_{0}=$ $k\left(1+\Delta_{\mathrm{nl}}\right)^{-1 / 3}$ corresponds to the pre-collapsed length scale of $k$. The parameter $\sigma_{8}$ is the rms linear mass fluctuation on $8 h^{-1} \mathrm{Mpc}$ scale at the redshift of interest, and $\beta=0.7+10 \Omega_{\nu}^{2}$. The functions $g_{0}=g\left(\Omega_{\mathrm{m}}, \Omega_{\Lambda}\right)$ and $g=g\left(\Omega_{\mathrm{m}}(a), \Omega_{\Lambda}(a)\right)$ are, respectively, the relative growth factor for the linear density field at present day and at $a$ for a model with a present-day matter density $\Omega_{\mathrm{m}}$ and a cosmological constant $\Omega_{\Lambda}$. A good approximation is given by 25

$$
g=\frac{5}{2} \Omega_{\mathrm{m}}(a)\left[\Omega_{\mathrm{m}}(a)^{4 / 7}-\Omega_{\Lambda}(a)+\left(1+\Omega_{\mathrm{m}}(a) / 2\right)\left(1+\Omega_{\Lambda}(a) / 70\right)\right]^{-1},
$$

and $\Omega_{\mathrm{m}}(a)=\Omega_{\mathrm{m}} a^{-1} /\left[1+\Omega_{\mathrm{m}}\left(a^{-1}-1\right)+\Omega_{\Lambda}\left(a^{2}-1\right)\right]$ and $\Omega_{\Lambda}(a)=\Omega_{\Lambda} a^{2} /[1+$ $\left.\Omega_{\mathrm{m}}\left(a^{-1}-1\right)+\Omega_{\Lambda}\left(a^{2}-1\right)\right]$. The time dependence is in factors $\sigma_{8}^{\beta}$ and $g$. For CDM and LCDM models, a good fit is given by $c_{1}=1.08 \times 10^{-4}$ and $c_{2}=2.10 \times 10^{-5}$. For C+HDM, a good fit is given by $c_{1}=3.16 \times 10^{-3}$ and $c_{2}=3.49 \times 10^{-4}$ for $\Omega_{\nu}=0.1$, and $c_{1}=6.96 \times 10^{-3}$ and $c_{2}=4.39 \times 10^{-4}$ for $\Omega_{\nu}=0.2$.

\subsection{High-Redshift Constraints}

The $\mathrm{C}+\mathrm{HDM}$ models discussed thus far are a class of models bridging the much-studied albeit troubled pure CDM and pure HDM models. They are parameterized by the neutrino density parameter $0<\Omega_{\nu}<1$, or equivalently, by the neutrino mass $0<m_{\nu}<93 h^{2} \mathrm{eV}$ (see Eq. (4)). The original motivation for examining these mixed models is to study whether the free-streaming effect 
introduced by the massive neutrinos could suppress the growth of density perturbations below the free-streaming scale, and thereby alleviate the problem of excess small-scale clustering in the standard CDM model. As illustrated in Figure 3, massive neutrinos do indeed reduce the amplitude of clustering at large $k$, and a larger $\Omega_{\nu}$ leads to smaller high $-k$ power.

Any viable cosmological model that provides a good statistical match to the local universe must also reproduce the appropriate evolutionary history out to high redshifts. Although gravitational clustering on galactic scales is indeed reduced in $\mathrm{C}+\mathrm{HDM}$ models, providing a better match to low-redshift observations 926 this suppression in the clustering power at high redshifts has been found to pose serious problems for some models. Studies based on semi analytic theories and dissipationless numerical simulations have shown 19.27 that flat C+HDM models with $\Omega_{\nu}>0.2$ do not produce enough early structure to explain the statistics of damped $\operatorname{Ly} \alpha$ systems at redshift $z \geq 2$. More recent work 28 has included the effects of gas ionization and dissipation in the theoretical calculations and has compared the results to new data for damped Ly $\alpha$ systems at even higher redshift $z \sim 4$. It is found that ionization of hydrogen in the outskirts of halos and gaseous dissipation near the halo centers tend to exacerbate the problem of late galaxy formation. The amount of dense gas associated with the damped systems falls well below that observed, even for the flat $\Omega_{\nu}=0.2 \mathrm{C}+\mathrm{HDM}$ model. This has placed an upper bound of $\sim 5$ $\mathrm{eV}$ on the sum of $\nu_{e}, \nu_{\mu}$, and $\nu_{\tau}$ masses, which is much more stringent than the upper limits given by current particle experiments (see Sec. 1).

Let us briefly discuss the limitations and uncertainties in these calculations. Still debated is the nature of damped Ly $\alpha$ absorption - whether it is due to intervening large, rapidly-rotating disk galaxies 29 with circular velocities $\gtrsim 200 \mathrm{~km} \mathrm{~s}^{-1}$, or infalling and randomly moving protogalactic gas clumps in dark matter halos 30 with virial velocities of $\sim 100 \mathrm{~km} \mathrm{~s}^{-1}$. When kinematic considerations are included, the latter model may have problems balancing the high energy dissipation rate caused by cloud collisions. 31 This uncertainty aside, in either scenario, a host dark halo of velocity at least $\sim 100 \mathrm{~km} \mathrm{~s}^{-1}$ is needed to reproduce the large velocity widths and asymmetries of the observed low-ionization lines associated with $\operatorname{Ly} \alpha$ systems. Uncertainties associated with the finite resolution of simulations have also been studied in some detail.32 It is found that even when the contribution from the numerically unresolved halos with velocities $v \lesssim 100 \mathrm{~km} \mathrm{~s}^{-1}$ is included, the absorption incidence is increased by at most a factor of 2 . This is insufficient to erase the discrepancies reported for flat $\mathrm{C}+\mathrm{HDM}$ models with $\Omega_{\nu}>0.2$. The upper bound on neutrino masses from current cosmological studies is therefore $\sim 5 \mathrm{eV}$. 


\section{Acknowledgments}

I thank the organizers P. Langacker and K. T. Mahanthappa of the TASI-98 School for their hospitality. This work was supported by the National Scalable Cluster Project at the University of Pennsylvania, the National Center for Supercomputing Applications, and a Penn Research Foundation Award.

\section{References}

1. See, e.g., Review of Particle Physics, Euro. Phys. Jour. C3, 1 (1998); T. Bowles, this volume.

2. See, e.g., A. Mann, this volume.

3. D. J. Fixsen, Ap. J. 473, 576 (1996).

4. R. Cowsik \& J. McClelland, Phys. Rev. Lett. 29, 751 (1972).

5. See, e.g., S. D. M. White, C. S. Frenk, \& M. Davis, Ap. J. Lett. 274, L1 (1983).

6. B. W. Lee \& S. Weinberg, Phys. Rev. Lett. 39, 165 (1977).

7. See, e.g., P. Langacker, this volume.

8. R. Bond \& A. Szalay, Ap. J. 274, 443 (1980).

9. C.-P. Ma, Ap. J. 471, 13 (1996).

10. E. M. Lifshitz, J. Phys. USSR 10, 116 (1946); E. M. Lifshitz \& I. M. Khalatnikov, Adv. Phys. 12, 185 (1963).

11. S. Weinberg, Gravitation and Cosmology (Wiley, New York, 1972); P. J. E. Peebles, The Large-Scale Structure of the Universe (Princeton University Press, Princeton, 1980).

12. H. Kodama \& M. Sasaki. Prog. Theo. Phys. Suppl. 78, 1 (1984); V. F. Mukhanov, H. A. Feldman, \& R. Brandenberger, Phys. Rep. 215, 206 (1992).

13. G. Efstathiou, in Physics of the Early Universe: Proceedings of the 36th Scottish Universities Summer School in Physics, ed. J. A. Peacock, A. E. Heavens, \& A. T. Davies (New York, Adam Hilger, 1990), p. 361; E. Bertschinger, Proceedings of Les Houches School, Session LX, ed. R. Schaeffer (Elsevier Science, Netherlands, 1995), p. 273; J. R. Bond, ibid., p.469.

14. C.-P. Ma \& E. Bertschinger, Ap. J. 455, 7 (1995).

15. C.-P. Ma \& E. Bertschinger. Ap. J. 429, 22 (1994).

16. J. Silk, Ap. J. 151, 459 (1968).

17. R. Bond, G. Efstathiou, \& J. Silk, Phys. Rev. Lett. 45, 1980 (1980).

18. D. Eisenstein \& W. Hu, Ap. J. 511, 5 (1998).

19. C.-P. Ma \& E. Bertschinger, Ap. J. Lett. 434, L5 (1994).

20. S. Tremaine, \& J. E. Gunn. Phys. Rev. Lett. 42, 407 (1979). 
21. A. J. S. Hamilton, P. Kumar, E. Lu, \& A. Matthews, Ap. J. Lett. 374, L1 (1991).

22. B. Jain, H. J. Mo, \& S. D. M. White, Mon. Not. Roy. Ast. Soc. 276, L25 (1995).

23. J. A. Peacock \& S. J. Dodds, Mon. Not. Roy. Ast. Soc. 280, L19 (1996).

24. C.-P. Ma, Ap. J. Lett. 508, L5 (1998).

25. O. Lahav, P. Lilje, J. Primack, \& M. Rees, Mon. Not. Roy. Ast. Soc. 251, 128 (1991); S. Carroll, W. Press, \& E. L. Turner, ARAA 30, 499 (1992).

26. See, e.g., A. Klypin, J. Holtzman, J. Primack, \& E. Regos, Ap. J. 416, 1 (1993); E. Gawiser \& J. Silk Science 280, 1405 (1998).

27. H. J. Mo \& J. Miralda-Escude, Ap. J. Lett. 430, L25 (1994); G. Kauffmann \& S. Charlot, Ap. J. Lett. 430, L97 (1994); A. Klypin, S. Borgani, J. Holtzman, \& J. R. Primack, Ap. J. 444, 1 (1995).

28. C.-P. Ma, E. Bertschinger, L. Hernquist, D. Weinberg, \& N. Katz, Ap. J. Lett. 484, L1 (1997).

29. J. Prochaska \& A. M. Wolfe, Ap. J. 487, 73 (1997).

30. M. Haehnelt, M. Steinmetz, \& M. Rauch, Ap. J. 495, 647 (1998).

31. McDonald, P., \& J. Miralda-Escudé, astro-ph 9809237 (1998).

32. J. P. Gardner, N. Katz, L. Hernquist, \& D. Weinberg, Ap. J. 484, 31 (1997). 